BULL. AUSTRAL. MATH. SOC.

MOS $12 \mathrm{~J} 20,13 \mathrm{~J} 10,16 \mathrm{~A} 80$

VOL. $5(1971), 425-426$.

\title{
Filtrations and valuations on rings
}

\author{
Helen E. Adams
}

The concept of a multiplicative filtration on a ring [4] is generalized so as to include among filtered rings, rings with valuation, pseudovaluation and semivaluation. The generalized filtration induces a topology on the ring, and the Hausdorff completion of the resulting topological ring [1, Chapters 1,2,3] is investigated as an inverse limit.

Next a generalized valuation and pseudovaluation on a ring are defined, and they are shown to induce a filtration on the ring which can then be completed. Conversely, a wide class of filtrations on a ring is shown to define pseudovaluations on the ring. And the completion of a ring with respect to another class of valuations is found to have no divisors of zero.

The rest of the thesis treats special cases of valuations: semivaluations on fields, and Manis [3] and Harrison [2] valuations on commutative rings. Results include that the completion of a (commutative) ring with respect to a Manis valuation is an integral domain, and necessary and sufficient conditions are obtained for the completion to be a field; the completion of a (commutative) ring with respect to a Harrison valuation is a field if the value group is archimedean ordered; and the completion of the rational numbers with respect to a semivaluation induced by the integers is a ring whose elements can be uniquely represented by expressions of the form $\sum_{i=1}^{\infty} i ! \alpha_{i}$ where $\alpha_{1} \in Q, 0 \leq \alpha_{1}<2$; and, for each $i \in I \backslash\{1\}, \alpha_{i} \in\{0,1, \ldots, i\}$.

Received 21 June 1971. Thesis submitted to Monash University, February 1971. Degree approved, June ${ }_{425}^{1971 .}$ Supervisor: Dr C.F. Moppert. 


\section{References}

[1] Nicolas Bourbaki, EZements of mathematics, General topology, Part 1 (Hermann, Paris; Addison-Wesley, Reading, Massachusetts; Palo Alto; London; Don Mills, Ontario; 1966).

[2] D.K. Harrison, "Finite and infinite primes in rings and fields", Mem. Amer. Math. Soc. 68 (Amer. Math. Soc., Providence, Rhode Island, 1966).

[3] Merle E. Manis, "Valuations on a commutative ring", Proc. Amer. Math. Soc. 20 (1969), 193-198.

[4] D.G. Northcott, Lessons on rings, modules and multiplicities (Cambridge University Press, Cambridge, 1968). 